\title{
Photoinduced optical anisotropy in organic molecular films controlled by an electric field
}

\author{
S.P. Palto ${ }^{a}$, L.M. Blinov ${ }^{b}$, S.G. Yudin ${ }^{a}$, G. Grewer ${ }^{c}$, M. Schönhoff ${ }^{c}$ and M. Lösche ${ }^{c}$ \\ a Institute of Organic Intermediates and Dyes, $103787 \mathrm{Moscow}$, Russian Federation \\ b Institute of Crystallography, Academy of Science, 117333 Moscow, Russian Federation \\ c Institute of Physical Chemistry, University of Mainz, W-6500 Mainz, Germany
}

Reccived 7 Scptcmber 1992; in final form 9 November 1992

The photoinduced reorientation of dye molecules in molecular films on solid substrates has been controlled with external dc electric fields, leading to solid state structures, which are macroscopically polar, temporally stable at room temperature, and welldefined at the molecular level. A simulation based on the excitation-driven rotational diffusion of the molecules in the potential of their neighbors yielded a realistic model and indicated a collective character of the process. This shows that we are close to a quantitative comprehension of the molecular interactions within these films.

\section{Introduction}

Photoinduced optical anisotropy (POA) has been shown to occur in a number of materials including polymers and Langmuir-Blodgett (LB) films [1]. In LB films, illumination with unpolarized light led to the decay of the polar order of asymmetrically prepared samples [2]. This was interpreted as a collective reorientation of chromophores following their optical excitation $[3,4]$. Here we report the first experiments in which the light-induced reorientation of molecules has been utilized to form macroscopically polar, stable solid state structures. Two examples for in-plane and for out-of-plane reorientation under the influence of static external fields perpendicular or parallel to the interface will be given, and a model based on the molecular interactions will be presented.

\section{Experimental}

The azobenzene derivative, dye 1 is weakly amphiphilic and has its ground state dipole moment, $\mu_{\mathrm{gr}} \approx 7.5 \mathrm{D}$, directed toward its hydrophilic end:

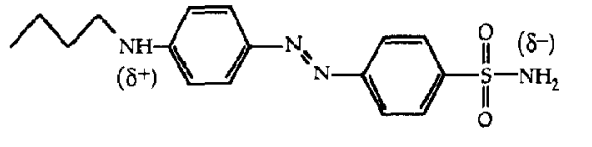

Compared to its non-substituted parent molecule, the absorption band with the highest extinction of 1 is shifted from the UV to the visible range of the spectrum (absorption maximum at $\lambda \approx 480 \mathrm{~nm}$ ) both in organic solvent, and in molecular films. The $\pi \rightarrow \pi^{*}$ and $n \rightarrow \pi^{*}$ transitions, which are separated in transazobenzene by more than $100 \mathrm{~nm}$, are no longer well distinguishable. The transition moment of the $\pi \rightarrow \pi^{*}$ transition is roughly oriented parallel to $\mu_{\mathrm{gr}}$. As determined by Stark spectroscopy, the excitation-induced dipole moment change of this transition is $\Delta \mu=4.5 \pm 0.2 \mathrm{D}$.

After deposition in a Langmuir film balance on an ultrapure $\mathrm{H}_{2} \mathrm{O}$ subphase from $\mathrm{CHCl}_{3}$ solution (area per molecule, $A \approx 40 \AA^{2}$ ), the molecules aggregate spontaneously, forming inhomogeneous films. Since the films did not sustain lateral pressure, they were only compressed until they appeared macroscopically homogeneous $\left(A \approx 25 \AA^{2}\right.$ per molecule). Subsequently, they were unidirectionally deposited on to hydrophobic solid substrates by repeatedly touching 
the aqueous interface horizontally (LangmuirSchaefer deposition). SAXS as well as ellipsometric characterization of the samples were consistent with an average thickness per deposited layer of $\approx 7 \AA$; the SAXS measurements indicated a large surface roughness of the samples.

Two samples configurations were prepared which enable the application of external electric fields in different orientations: (a) 10 molecular layers were deposited on a quartz slide bearing microfabricated $\mathrm{Cr}$ electrodes, which interdigitate at a lateral distance of $10 \mu \mathrm{m}$, to apply a field parallel to the interface [5]. (b) Alternatively, 13 layers were deposited on a indium-tin oxide (ITO) covered glass slide, which was precoated with 5 layers of arachidic acid; 4 layers of arachidic acid were subsequently deposited for insulation prior to evaporating $\approx 100 \AA$ of $\mathrm{Al}$ as a counter electrode ("sandwich sample configuration", total thickness of the organic layer system $\approx 310 \AA$ ). This configuration permits field application parallel to the film normal, $z$. These electrode configurations were used to apply electric fields both for sample characterization in Stark spectroscopic experiments and for photoelectro-poling.

Stark spectroscopy has been dealt with in numerous treatises [6-8]. The absorption change, $\Delta A$, due to an electric field at the site of the chromophore, $F$, depends on molecular properties of the chromophores ( $\Delta \mu$ : change of the dipole moment and $\Delta \alpha$ : change of polarizability on excitation) and on their orientation with respect to $F(\theta$ : angle between $F$ and $\Delta \mu)$ :

$$
\begin{aligned}
\Delta A & =\frac{\Delta \mu\langle\cos \theta\rangle}{h c} \tilde{\nu} \frac{\partial}{\partial \tilde{\nu}}\left(\frac{A}{\tilde{\nu}}\right) F+\frac{1}{2} \frac{\Delta \alpha}{h c} \tilde{\nu} \frac{\partial}{\partial \tilde{\nu}}\left(\frac{A}{\tilde{\nu}}\right) F^{2} \\
& +\frac{1}{2} \frac{\Delta \mu^{2}\left\langle\cos ^{2} \theta\right\rangle}{h^{2} c^{2}} \tilde{\nu} \frac{\partial^{2}}{\partial \tilde{\nu}^{2}}\left(\frac{A}{\tilde{\nu}}\right) F^{2} \\
& =c_{1} F+\left(c_{2}+c_{3}\right) F^{2} .
\end{aligned}
$$

The angular brackets denote macroscopic averages over the sample, $\tilde{v}$ is the wave number, $h$ is Planck's constant and $c$ is the light velocity. Experimentally, the response of the energy spectrum, $\triangle A$, of the chromophores to a sinusoidal external electric field, $\boldsymbol{F}_{\mathrm{ac}}$, is revealed by phase sensitive detection of changes in the transmittance of the sample. Different contributions to the Stark effect are separated by harmonic discrimination by exploiting their distinct dependencies on $\boldsymbol{F}_{\text {ac. }}$. It is assumed that reorientation of dye molecules due to the field modulation and contributions of higher order terms in $F_{\text {ac }}$ may be neglected. The evaluation of the spectra may be aggravated by intrinsic or external static electric fields, $F_{\text {int }}$ or $F_{\text {ext }}$, which lead to a linearization of the quadratic terms, i.e. to a pseudo-linear effect $[7,9]$ :

$$
\begin{aligned}
\Delta A & =c_{1} F_{\mathrm{ac}}+2\left(c_{2}+c_{3}\right)\left(F_{\mathrm{int}}+F_{\mathrm{ext}}\right) F_{\mathrm{ac}} \\
& +\left(c_{2}+c_{3}\right) F_{\mathrm{ac}}^{2} .
\end{aligned}
$$

Absorption and Stark spectra were measured at 300 $\mathbf{K}$ in a spectrometer of local design [5] with amplitudes of $F_{\mathrm{ac}} \approx 330 \mathrm{kV} / \mathrm{cm}$ or $\approx 50 \mathrm{kV} / \mathrm{cm}$ for the sandwich and interdigitated electrode configurations, respectively, and with a modulation frequency of $1 \mathrm{kHz}$. The photo-reorientation experiments were conducted with a Xe lamp (power density within the long wavelength absorption band of the dye, 400 $\mathrm{nm}<\lambda<600 \mathrm{~nm}: 40 \mathrm{~mW} / \mathrm{cm}^{2}$, unpolarized; $18 \mathrm{~mW} /$ $\mathrm{cm}^{2}$, polarized). The probing light intensity of the spectrometer was three orders of magnitude lower. A Glan polarizer was used where appropriate.

\section{Results}

\subsection{In-plane field application}

Absorption spectra of molecular film samples of 1 (see fig. 1a) were identical to those published earlier [2]. Freshly prepared samples on interdigitated electrodes were not ideally isotropic within the film area. This is seen from a small but detectable linear Stark effect in fig. $1 \mathrm{~b}$, spectrum 1 . Its magnitude and sign depended on the location within the sample area where the spectrum was taken. This is an indication that the films are organized in macroscopic domains with different average orientations of their in-plane dipole moment components.

On application of an in-plane poling field under illumination polarized along the $\boldsymbol{x}$ direction ( $\boldsymbol{x}$ : unit vector parallel to the interdigitating electrodes) the linear Stark effect increased dramatically, despite a concomitant decrease of the absorbance by $32 \%$, see spectrum 2 in fig. $1 \mathrm{~b}$ (poling field: $F_{\mathrm{dc}}^{y}=+55 \mathrm{kV} /$ $\mathrm{cm}$; $5 \mathrm{~min}$ illumination). The quadratic Stark spec- 

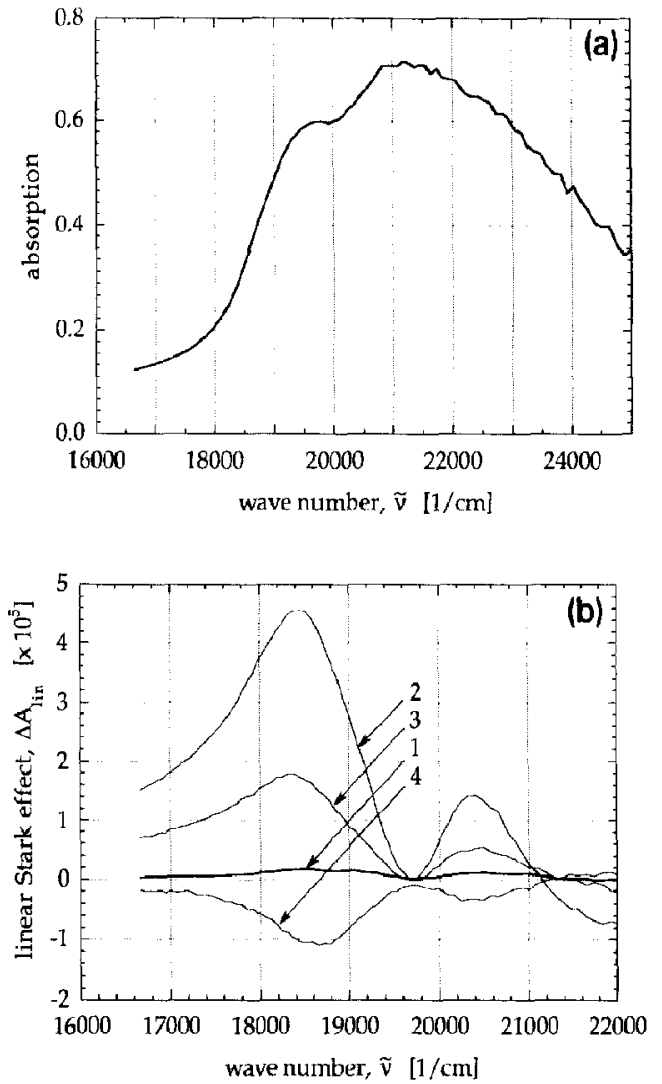

Fig. 1. (a) Absorption spectrum of a sample of 1 (13 polar layers of the dye plus 4 layers of $C d$ arachidate, thickness $d \approx 310 \AA$ ) on glass. (b) Development of the in-plane order parameter on photoelectropoling within the plane of the molecular films (interdigitated electrodes). The linear Stark spectra, $\Delta A_{\text {lin }}$, after the poling have been corrected for decreases in their absorptions due to the illumination, in order to enable comparison of the products $\Delta \mu \mathrm{F}_{\mathrm{ac}}\langle\cos \theta\rangle$. The amplitudes of $F_{\mathrm{ac}}$ were $50 \mathrm{kV} / \mathrm{cm}$ in all cases. (1) Freshly prepared sample. Magnitude and polarity of the Stark effect change irregularly across the sample arca. (2) After 5 min of polarized illumination $(\| x)$ under an in-plane static electric field $(\| y), F_{\mathrm{dc}}^{y}=55 \mathrm{kV} / \mathrm{cm}$; correction factor compensating the decrease in optical density of the sample on illumination, $c=1.49$. (3) After subsequent polarized illumination $\| y$ (25 min, no poling field); $c=2.67$. (4) After subsequent polarized illumination $\| x(15 \mathrm{~min})$ with the poling field anti-parallel to the situation in (2), $F_{\mathrm{dc}}^{y}=-55 \mathrm{kV} / \mathrm{cm} ; c=3.47$. The scale on the ordinate corresponds to spectrum (1).

trum remained almost unaffected (a decrease in magnitude by $\approx 15 \%$ occurred). The shape of the quadratic spectrum was proportional to the derivative of the linear spectrum, as predicted by eq. (1) if the second term is small compared to the third.
This suggests that the Stark spectra are dominated by the interaction of $\boldsymbol{F}_{\mathrm{ac}}$ with $\Delta \boldsymbol{\mu}$.

A subsequent illumination ( $25 \mathrm{~min}$ ) with light polarized along $y$ and without a poling field led to a decay of the linear Stark effect (spectrum 3 in fig. lb) while its sign was maintained. Further illumination ( $15 \mathrm{~min}$ ) polarized along $\boldsymbol{x}$ under $F_{\mathrm{dc}}^{y}=-55 \mathrm{kV} / \mathrm{cm}$ led to a reversal of the linear Stark effect (spectrum 4 in fig. 1b), indicating an average orientation of the $y$ components of the domain directors which opposes those of the former states. Fig. Ib visualizes the relative changes in the lateral order parameter $\langle\cos \theta\rangle$, since decreases in the absorption of the sample (due to a slight tendency of the chromophores to evade towards the sample normal) have been compensated for by multiplication of the raw data with the appropriate correction factors, cf. eq. (1).

Our interpretation of these results is that a rotational reorientation of the excited molecules occurs. The direction of this reorientation is biased by the application of the static external field during illumination. Thus electro-poling during illumination leads not only to optical anisotropy in the samples but even to macroscopic polarity of the sample in the substrate plane. Without illumination this polarity is stable on the time scale of months at room temperature.

\subsection{Out-of-plane field application}

In sandwich samples, on illumination with polarized light $(5 \mathrm{~min}$ ) the optical density for unpolarized light decreased by $\approx 20 \%$, independent of the application, and of the direction, of a dc poling field parallel to $z\left(F_{\mathrm{dc}}^{z}=+1.65\right.$ or $\left.-1.65 \mathrm{MV} / \mathrm{cm}\right)$. Similarly, changes in the Stark spectra after illumination were independent of $F_{\mathrm{dc}}^{z}$. Due to the polar organization of the film, the magnitude of the linear Stark effect was larger than that of the quadratic effect by more than two orders of magnitude.

In contrast, the application of a $\mathrm{dc}$ poling field during illumination with unpolarized light extended a pronounced effect on the absorption and Stark spectra. This indicates a lower activation energy of in-plane reorientation, which is induced by polarized light as shown above, but is not detected in the sandwich sample geometry, than that of out-of-plane 
reorientation, which is driven with a significant efficiency only by unpolarized light. On illumination $(5 \mathrm{~min})$ under a field $F_{\mathrm{dc}}^{z}=+2.55 \mathrm{MV} / \mathrm{cm}$ with respect to the ITO electrode, a decrease in absorbance by $\approx 35 \%$ was observed, whereas at $F_{\text {dc }}^{z}=-2.55$ $\mathrm{MV} / \mathrm{cm}$ a drop by $\approx 75 \%$ occurred. As in fig. $1 \mathrm{~b}$, the linear Stark spectra, fig. 2, are corrected for these decreascs to cnable direct comparison of the order parameters.

The increase of the corrected Stark spectrum on photoelectro-poling under $F_{\mathrm{dc}}^{z}>0$ indicates that the macroscopic polar order of the films has been increased, compare spectra 1 and 2 in fig. 2 . If the direction of the poling field is parallel to the polar order of the freshly prepared $\left(F_{\mathrm{dc}}^{z}<0\right)$, which leads to spectrum 3 in fig. 2 , the situation is more complicated: It has been shown that reorientation of dipolar molecules in LB films generally leads to a change in the static electric field, $F_{\text {int }}$, within the sample [10]. This is responsible for the very different changes in the linear Stark spectra, both in shape and amplitude, on poling with $F_{\mathrm{dc}}^{z}>0$ or $F_{\mathrm{dc}}^{z}<0$. Specifically the latter leads to the formation of an internal field which augments the negative poling field and, at the same time, contributes a component to the spectrum, that originates from the quadratic effect, cf. eq. (2). This pseudo-linear contribution is small compared to the linear contribution from the

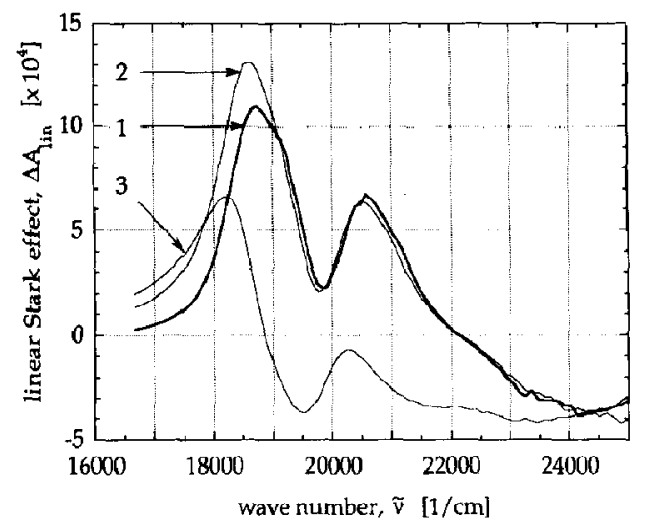

Fig. 2. Linear Stark spectra of a polar sandwich sample of 1 (13 dye layers, total thickness with 9 insulation layers, $d \approx 310 \AA$ ). (1) Freshly prepared sample. (2) After $5 \mathrm{~min}$ of illumination with unpolarized light under a dc electric field opposing the polar orientation of the molecules $\left(F_{\mathrm{dc}}^{z}>0\right) ; c=1.54$. (3) Same as (2), but opposite poling field polarity, $F_{\mathrm{dc}}^{z}<0 ; c=3.85$. The scale on the ordinate corresponds to spectrum (1). intrinsic Stark effect in the case of the ordered polar structure, but large after reorientation under $F_{\mathrm{dc}}^{z}<0$ (note that spectrum 3 in fig. 2 has been multiplied by $\approx 3.8$ to compensate for a reduction in optical density by $74 \%$ !). In the latter case, molecules which reorient into the direction of the poling field must cross the direction of the electric field vector of the illumination. Consequently, a large portion of the molecules misalign with respect to the poling field direction, resulting in a small intrinsic Stark effect but a large reduction in optical density.

$F_{\text {int }}$ can be estimated from a comparison of the linear and quadratic (data not shown) Stark spectra of the sample poled with $F_{\mathrm{dc}}^{z}<0$ : At a wave number, $\tilde{\nu}_{0} \approx 22150 \mathrm{~cm}^{-1}$, where $\Delta A_{\text {lin }}=0$ in the native sample, the intrinsic linear effect vanishes, and the quadratic effect is exclusively determined by the interaction of $\boldsymbol{F}_{\mathrm{ac}}$ with $\Delta \boldsymbol{\mu}$, see eq. (1). Evaluation of eq. (2) after separation of the linear and quadratic contributions then leads to

$F_{\mathrm{int}}=\frac{1}{2} \frac{\Delta A_{\mathrm{lin}}(\tilde{\nu})}{\Delta A_{\mathrm{q}}(\tilde{\nu})} \frac{F_{\mathrm{ac}, \mathrm{q}}^{2}}{F_{\mathrm{ac}, \mathrm{lin}}}$

$$
\text { ( } \approx 10 \mathrm{MV} / \mathrm{cm} \text { after poling with } F_{\mathrm{dc}}^{z}<0 \text { ). }
$$

The difference between two linear Stark spectra of the poled sample measured under the influence of different external dc fields, $F_{\text {ext }}$ (data not shown), is used to determine the pseudo-linear contribution of $F_{\text {int }}$ to the spectrum, cf, fig. 3: Subtraction of this

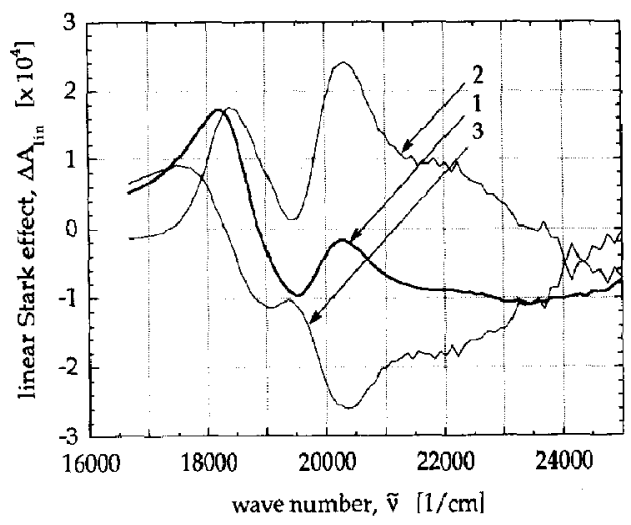

Fig. 3. Correction of the linear Stark spectrum, trace (3) of fig. 2 , for the contribution of a static electric field in the sample. (1) Uncorrected spectrum. (2) Internal field contribution. (3) The corrected linear Stark spectrum indicates that the polarity of the sandwich sample has been reversed. Details see text. 
contribution (spectrum 2) from the original Stark spectrum (1) yields the intrinsic linear spectrum (3). A comparison with the Stark effect of the sample prior to poling, spectrum 1 in fig. 2, indicates that the initial polarity of the film structure has been inverted in the reorganization.

\section{Simulation}

The absorption changes in illumination with intense light have been attributed to a collective reorientation of two-dimensional domains of the molecules, with a rotation of their directors away from the electric field vector of the excitation light [2]. The net result after a large number of excitations is a depletion of transition moments parallel to the field vector of the exciting light, and the change in molecular orientation is indeterminate, so that the final orientation distribution is symmetric around the depleted direction. This symmetry is broken by the external field. The surprising observation that an interaction energy of the field with an individual molecule, $\boldsymbol{F} \cdot \boldsymbol{\mu}_{\mathrm{gr}} \approx 1 \mathrm{meV}$, much lower than the thermal energy is sufficient to control the reorientation process is another indication of its collective character.

Based on this model, we have simulated the influence of an external electric field on the reorientation of molecules within one macroscopic domain. In a spherical coordinate system $(\vartheta, \varphi)$ these molecules populate $2 N \times N$ states on a discrete angular lattice $[3,4]$ in a potential

$$
\begin{aligned}
& W=A_{2} S_{2}\left(\frac{3}{2} \sin ^{2} \psi-1\right)-A_{1} S_{1} \cos \psi \\
& +\mu_{\mathrm{gr}} \cdot \frac{\boldsymbol{P}_{1}}{\boldsymbol{\epsilon}_{0}}-\boldsymbol{\mu}_{\mathrm{gr}} \cdot \boldsymbol{F}_{\mathrm{loc}} .
\end{aligned}
$$

$\psi$ is the angle between the long axis of a molecule and the domain director. The unpolar orientational part of the interaction energy has the form of a nematic Maier-Saupe potential with an order parameter $S_{2}=\frac{1}{2}<3 \cos ^{2} \psi-1>$. The second contribution accounts for the polarity of the sample with a characteristic interaction energy $A_{1}$ and a polar order parameter $S_{1}=\langle\cos \psi\rangle$. The third and fourth terms describe the dipole interactions with the polarization field ( $\boldsymbol{P}_{1}$ : polarization of the domain) and the external field $\boldsymbol{F}_{\text {loc. }}$.

A discrete reorientation step, either thermally or optically induced, has a probability $P$ to drive a molecule into a state $(\vartheta, \varphi)$ which depends on $W$ :

$P(\theta, \varphi)=c_{0} \exp \left(-\frac{W(\vartheta, \varphi)-W_{0}}{k_{\mathrm{B}} T}\right)$,

where $W_{0}=-A_{2} S_{2}-A_{1} S_{1}+\mu_{8 \mathrm{r}} P_{1} / \epsilon_{0}+\mu_{\mathrm{gr}} e_{1} \cdot F_{\text {loc }}$ is the ground state energy $\left(e_{1}\right.$ : unit vector of the director orientation, $c_{0}$ : normalization constant).

Thermal equilibrium is defined by a balance of fluxes into and form $(\vartheta, \varphi)$. Hence the normalized population density of the state, $f(\vartheta, \varphi)$, is constant in time:

$B[P(\vartheta, \varphi)-f(\vartheta, \varphi)]=0$.

$B=1 / \tau$ is the relaxation rate to thermal equilibrium. Illumination polarized along $(\pi / 2,0)$ destroys this equilibrium and establishes a net anisotropic flux

$$
\begin{aligned}
& \frac{\partial f(\vartheta, \varphi)}{\partial t}=B[P(\vartheta, \varphi)-f(\vartheta, \varphi)] \\
& \quad+K\left[P(\vartheta, \varphi) G-f(\vartheta, \varphi) \sin ^{2} \vartheta \cos ^{2} \varphi\right],
\end{aligned}
$$

where

$G=\iint f\left(\vartheta^{\prime}, \varphi^{\prime}\right) \sin ^{3} \vartheta^{\prime} \cos ^{2} \varphi^{\prime} \mathrm{d} \vartheta^{\prime} \mathrm{d} \varphi^{\prime}$

and $K=n \sigma$ is the product of photon flux and absorption cross section of the chromophore.

Results of a simulation of in-plane reorientation are shown in fig. 4 . Both the light polarization and field were parallel to the substrate and perpendicular to each other. The power density was $Q=n h c=5$ $\mathrm{mW} / \mathrm{cm}^{2} . F_{\mathrm{dc}}^{y}= \pm 10^{6} \mathrm{~V} / \mathrm{cm} ; \mu_{\mathrm{gr}}=3 \mathrm{D} ; \sigma=10^{-17}$ $\mathrm{cm}^{2} ; N=72$. The magnitudes of $A_{2}=0.16 \mathrm{eV}$ and $A_{1}=0.3 \mathrm{eV}$ were chosen so that a domain was thermally stable at $T=300 \mathrm{~K}$. The value $\tau=60 \mathrm{~s}$ is the experimentally observed relaxation time to thermal equilibrium from a new director orientation when the illumination is turned off [1]. The orientation distribution of the domain in thermal equilibrium with its initial director orientation at $(\vartheta, \varphi)=\left(75^{\circ}\right.$, $\left.10^{\circ}\right)$, fig. $4 a$, has its director at $\left(57.5^{\circ}, 87.5^{\circ}\right)$ after $450 \mathrm{~s}$ of irradiation attracted by $F_{\mathrm{dc}}^{y}>0\left(\varphi_{\mathrm{F}}=90^{\circ}\right)$, fig. $4 \mathrm{~b}$. A moderate evasion toward the interface nor- 
a)
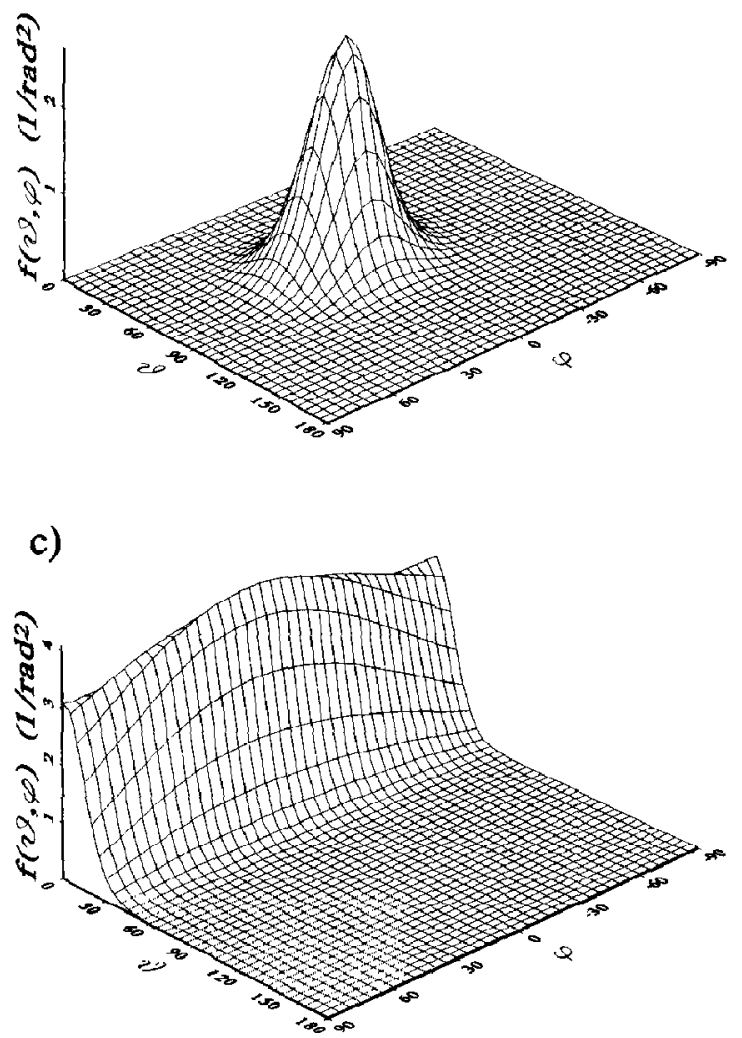

b)
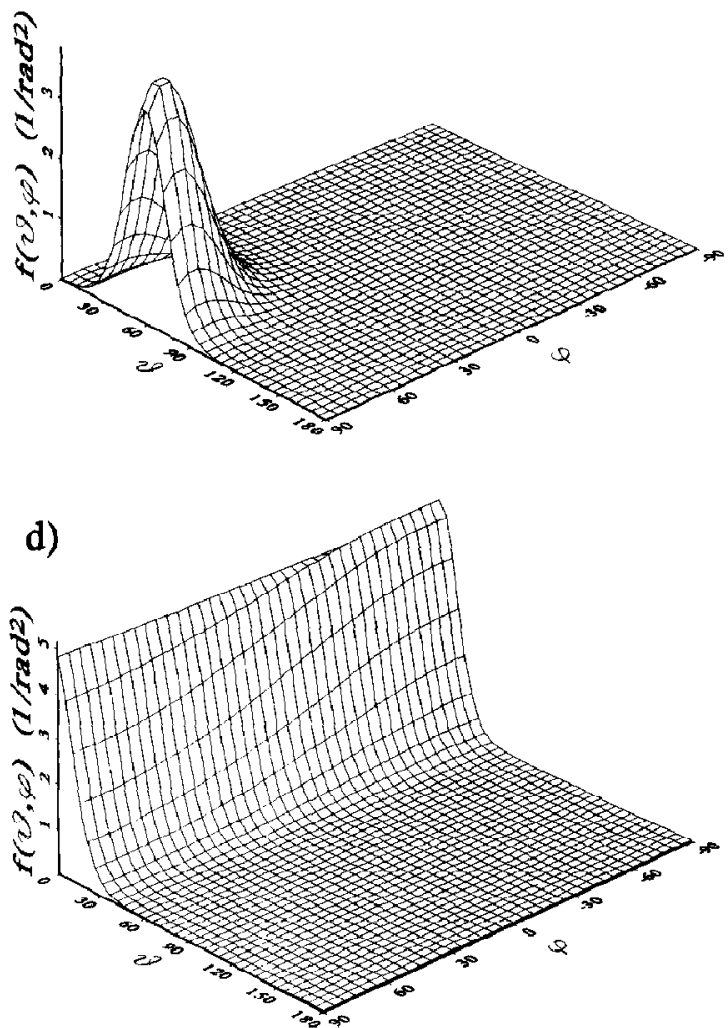

Fig. 4. Simulated time development of the orientational distribution function $f(\vartheta, \varphi)$ of chromophores in a domain, projected on a square lattice. A thermally equilibrated distribution from a state $\left(\theta=75^{\circ}, \varphi=10^{\circ}\right),(\mathrm{a})$, is shown after $450 \mathrm{~s}$ of illumination polarized along $\varphi=0^{\circ}$ with a poling field oriented at $\varphi_{F}=90^{\circ}$ (b), or at $\varphi_{F}=-90^{\circ}$ (c) 450 s and (d) 1100 of illumination, respectively). For details, see text.

mal (leading to only a slight decrease in absorbance) is found. In contrast, if attracted by $F_{\mathrm{dc}}^{y}<0$ $\left(\varphi_{F}=-90^{\circ}\right)$ from the same initial situation, the molecular orientations evade collectively toward the film normal (fig. 4c; director at $\left(10^{\circ}, 10^{\circ}\right)$ at $t=450 \mathrm{~s}$ ), thus avoiding the direction of the light polarization at $\left(90^{\circ}, 0^{\circ}\right)$, before they are redirected toward the poling field (fig. $4 \mathrm{~d}$; director at $\left(5^{\circ},-87.5^{\circ}\right)$ at $t=1$, $100 \mathrm{~s}$ ).

\section{Discussion}

The results of the simulation reflect many of the experimental observations quantitatively or qualitatively, while all parameters are either directly de- rived from the experimental situation or correspond to typical interaction coefficients: The simulations show that a minimum value of the potential $A_{1} \approx 0.3$ $\mathrm{eV}$ is required to stabilize a parallel alignment of ncighboring dipoles over an anti-parallel alignment. This value corresponds roughly to the energy of one hydrogen bond. Structurally, a molecule of 1 may form three hydrogen bonds to its nearest neighbors in a parallel alignment, whereas only two can be formed if the chromophores align anti-parallel. In this context it is interesting to note that photo-reorientation was not observed in experiments with azobenzenes on which the amine substituent has been replaced by an oxygen.

The minimal interaction energy necessary to control the reorientation process in the simulation was 
found to be $\boldsymbol{F} \cdot \boldsymbol{\mu}_{\mathrm{gr}} \geq 0.5 \mathrm{meV}$ (slightly dependent on the initial director orientation with respect to light polarization). This is three orders of magnitude smaller than the potentials between the interacting chromophores and is in quantitative accord with the experimental observations. Finally, the observation that the domain director avoids crossing the polarization direction of the illumination (compare figs. $4 \mathrm{~b}$ and $4 \mathrm{c}$ ) resembles the experimental situation, where a pronounced asymmetry in the temporal evolution of the optical properties has been observed on switching the polarity of the poling field on a sandwich sample, see fig. 2. A quantitative comparison of the order parameters in experiments and simulation, however, is currently not feasible, since the results from the simulation refer to one domain of molecules (and depend on the initial director orientation), whereas the spectroscopic result is a macroscopic average.

It is, at point, difficult to decide whether or not trans-cis-trans isomerizations are instrumental in inducing rotations of the chromophores. In the discussion of photo-orientation in polymeric and in liquid-crystalline samples, isomerization reactions are generally held responsible for the observed reorganization phenomena [11-15]. On the other hand, it has been shown that the trans-cis isomerization is effectively suppressed in Langmuir films if the chromophores are organized in closely packed aggregates [16]. AFM as well as electron diffraction experiments indicate that the chromophores are indeed organized in highly ordered molecular aggregates in our samples [17]. We emphasize that the simple assumption of thermalization of the excitation energy is sufficient to explain our experimental results. Our model for the collective reorientation does not depend on the nature of the primary photoprocess which leads to the rotational reorganization. We will address the question about the molecular mechanism and the role of isomerization reactions in the process in future work.

The correspondence of the simulated results with the experimental observations demonstrates that we are well on our way to comprehend the reorientation process on a molecular level. Thus a controlled manipulation of stable, macroscopically polar solid state structures in the form of molecularly well-defined films for advanced applications seems feasible.

\section{Acknowledgement}

We thank H. Möhwald, Mainz, for his support of this work.

\section{References}

[1] M.I. Barnik, V.M. Kozenkov, N.M. Shtykov, S.P. Palto and S.G. Yudin, J. Mol. Electron. 5 (1989) 53, and references herein.

[2] M.I. Barnik, S.P. Palto, V.A. Khavrichev, N.M. Shtykov and S.G. Yudin, Thin Solid Films 179 (1989) 493.

[3] S.P. Palto, V.A. Khavrichev, S.G. Yudin, L.M. Blinov and A.A. Udal'yev, Mol. Mater. 2 (1992), in press.

[4] S.P. Palto, M.I. Barnik, V.A. Khavrichev, N.N. Davydova and S.G. Yudin, Thin Solid Films (1992), in press.

[5] G. Grewer and M. Lösche, Makromol, Chem. Macromol. Symp. 46 (1991) 79.

[6] W. Liptay, in: Excited states, ed. E.C. Lim (Academic Press, New York, 1974) p. 129.

[ 7] R. Reich and S. Schmidt, Ber. Bunsenges. Physik. Chem. 76 (1972) 589 .

[8] L.M. Blinov, Sov. Sci. Rev. A 12 (1989) 1.

[9]G. Grewer and M. Lösche, Thin Solid Films 210/211 (1992) 670 .

[10] L.M. Blinov, S.P. Palto, A.A. Udal'yev and S.G. Yudin, Thin Solid Films 179 (1989) 351.

[11] J.H. Wendorff and M. Eich, Mol. Cryst. Liquid Cryst. 169 (1989) 133.

[12] U. Wiesner, N. Reynolds, C. Boeffel and H.W. Spiess, Liquid Cryst. 11 (1992) 251.

[13] S. Ivanov, L. Yakovlev, S. Kostromin, V. Shibaev, L. Lasker, J. Stumpe and D. Kreysig, Makromol. Chem. Rapid Commun. 12 (1991) 709.

[14] S. Hvilsted, F. Andruzzi and P.S. Ramanujam, Opt. Letters 17 (1992) 1

[15] A. Natansohn, S. Xie and P. Rochon, Macromolecules 25 (1992) 5531.

[16] M. Iwamoto, Y. Majima, H. Naruse and K. Iriyama, J. Appl. Phys. 72 (1992) 1631.

[17] M. Schönhoff, G. Grewer, L.-F. Chi, H. Fuchs and M. Lösche, unpublished results. 